(C2019, Elsevier. Licensed under the Creative Commons Attribution-NonCommercialNoDerivatives 4.0 International http://creativecommons.org/about/downloads

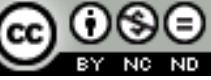




\title{
Application of Pure Shift and Diffusion NMR for the characterisation of crude and processed pyrolysis oil.
}

\author{
Adam LE GRESLEYa*, Grace BROADBERRYa, Cameron ROBERTSONª , Jean-Marie \\ R. PERONa , Joshua ROBINSON ${ }^{a}$ and Shane O'LEARY ${ }^{b}$
}

aDepartment of Chemical and Pharmaceutical Sciences, Faculty of SEC, Kingston University, Kingston-upon-Thames, Surrey, KT1 2EE, UK.

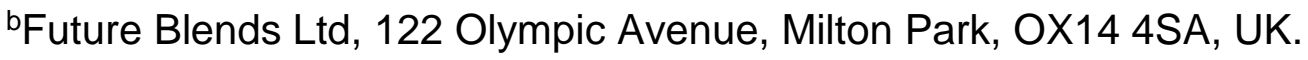

${ }^{*}$ Corresponding author:

Dr Adam Le Gresley, Tel + 44 (0)20 84177432 Email: a.legresley@kingston.ac.uk

\section{Highlights}

Diffusion NMR used to characterise molecular mass distribution of pyrolysis oil components.

PSYCHE-iDOSY experiments acquired on pyrolysis oil for the first time.

Semi-automatic characterisation of water-soluble components using Chenom ${ }^{\mathrm{TM}}$

\begin{abstract}
By combining diffusion-ordered NMR spectroscopy (DOSY) and pure shift NMR spectroscopy ((Pure Shift Yielded By Chirp Excitation (PSYCHE)) with 1D NMR metabolite assignment, we demonstrate an improved method for in situ analysis of pre and post-processed pyrolysis oil to quickly establish the most effective upgrading
\end{abstract}


procedure. These experiments use molecular mass estimations from DOSY and single component verification using PSYCHE to confirm the identity of metabolites and show how the mass pattern for pyrolysis oils varies depending on how it is upgraded.

With the use of a semi-automatic approach for metabolite assignment, we have verified and quantified individual components, giving rise to a collection of potential 'marker compounds'; their changes in concentration being correlated to the upgrading process a pyrolysis oil undergoes.

Keywords: Complex mixture analysis, NMR, Pure shift NMR, Diffusion-Ordered Spectroscopy, PSYCHE-iDOSY

\section{Introduction}

\subsection{Pyrolysis oil composition}

Previous studies have shown that by varying both the organic feedstock and pyrolysis conditions, the resultant oils can have vastly different properties. Predicated on their consisting of a wide range of compounds, including sugars,carbohydrates, furans, ketones, aldehydes and carboxylic acids in varying quantities..$^{1-4}$

The high levels of oxygenated hydrocarbons and water cause delayed ignition and low heating values.Due to the presence of carboxylic acids, bio-oils can be highly acidic and corrosive. ${ }^{5,6}$

Pyrolysis oils are also very viscous and chemically unstable and have been found to degrade over time. Phase separation and reduced volatility are often observed as a result of the ageing of the oil. ${ }^{7,8,9}$ The instability of pyrolysis oils can mainly be explained by the presence of aldehydes, ketones, and sugars of the feedstock. The 
main factor however has been suggested to be high molecular weight lignin oligomers/derivatives produced from the pyrolysis process ${ }^{10}$

It is, therefore, necessary for crude bio-oil to undergo upgrading prior to use as a fuel. Methods include hydrotreatment, hydrocracking, transesterification, and ketonisation. Depending on the upgrading process an oil undergoes, its chemical components and their relative concentrations will vary. ${ }^{11,12}$ Refinement techniques are usually aimed at reducing the oxygen and carboxylic acid content, whilst increasing the long chain hydrocarbon concentration to increase the volatility and energy density of the fuel. ${ }^{13}$ Unstable compounds are primarily eliminated by conversion of aldehydes, ketones and sugars to alcohols through hydrogenation and methods used for separation of high molecular weight lignin derivatives from the oil. ${ }^{10}$

Extensive research has been made into the effects of varying the pyrolysis temperature and the biomass feedstock on the composition of the resulting oils, however, there is limited data that compares the effects of different upgrading techniques on the pyrolysis oils. This is due to the difficulty in analysing these samples in situ. None of the standard techniques such as High Performance Liquid Chromatography (HPLC) or Liquid Chromatography-Mass Spectrometry (LC-MS) is particularly well suited, owing to the need to optimise extraction for each class of pyrolysis oil components and the characteristics of the oils requiring multiple extraction and purification steps to enable the successful use of a chromatographic separation technique.

1.2 General NMR analysis of pyrolysis oils 
Previous work by Hao et al. ${ }^{5}$ and others, ${ }^{7},{ }^{14}$ details the use of NMR spectroscopy to characterise pyrolysis oils using ${ }^{1} \mathrm{H},{ }^{13} \mathrm{C},{ }^{31} \mathrm{P},{ }^{19} \mathrm{~F}$, and Heteronuclear Single Quantum Correlation spectroscopy (HSQC) experiments. Using 1D proton NMR alone to identify single compounds within a pyrolysis oil mixture is a challenging task.

Due to some peaks appearing across a wide range of chemical shifts (hydrogen bonding in polar solvents can cause hydroxyl groups' signal to shift widely), there are extensive limitations to analysing quantitatively bio-oils using ${ }^{1} \mathrm{H}$ or ${ }^{13} \mathrm{C}$ NMR alone. Previous literature details the successful identification of single sugars and aromatics such as xylose, guaiacol and stilbene using HSQC experiments. ${ }^{14}$

HSQC is a helpful method for the separation of protons with very similar chemical shifts, which are bonded to different carbon atoms. However, when dealing with such complicated mixtures, poor resolution of peaks due to extensive overlapping can make even HSQC spectra of pyrolysis oils difficult to analyse.

The main aim of this study was to investigate whether a combination of Diffusion Ordered Nuclear Magnetic Resonance Spectroscopy (DOSY) and Pure Shift Yielded by Chirp Excitation (PSYCHE) could be used as an improvement on existing methods for the NMR characterisation and quantitation of pyrolysis oils following various upgrading processes. The objectives of this project were to use a selection of one, two and three-dimensional NMR methodologies to give spectra which are characteristic of a particular upgrading procedure and elucidate the structures of single components present in the oils for comparative studies.

\subsection{DOSY NMR}


DOSY NMR spectroscopy is a pseudo-2D technique that gives virtual separation of proton environments based on their diffusion coefficient.

The size of the 2D contours in a DOSY spectrum reflects the standard error. In complex mixtures with poorly resolved proton spectrum, the DOSY spectrum can have multiple overlapping signals making separation poor in highly concentrated areas such as the aliphatic region of pyrolysis oils. High Signal to Noise $(\mathrm{S} / \mathrm{N})$ peaks in the proton dimension such as 3-(Trimethylsilyl)propionic acid-d4 sodium salt TSP (Oppm), and DMSO (2.5ppm) are very small contours on the DOSY spectrum, with minimal error in the diffusion coefficient.

\subsection{Pure Shift}

Pure Shift NMR spectroscopy, also known as broadband homodecoupling, is not new, but it has recently been gaining more focus and is currently being applied in an array of combined NMR methods. ${ }^{15}$

The purpose of pure shift NMR is to suppress ${ }^{1} \mathrm{H}-{ }^{1} \mathrm{H}$ coupling and hence collapse all multiplets into singlets. Consequently, this leads to a large improvement in the resolution and vastly reduces signal overlap. This is a very attractive NMR method for the characterisation of complex mixtures with an abundance of peaks in the 1D

${ }^{1} \mathrm{H}$ spectra. Long experiment time and inconsistent coupling suppression have hindered the use of pure shift NMR in complex mixture analysis. $16,17,18,19,20$ The most recent advance in pure shift NMR and the one that is implemented in this study is known as Pure Shift Yielded by Chirp Excitation (PSYCHE). Two small flip angle swept-frequency pulses are used in combination with a weak pulsed field gradient to achieve the homonuclear decoupling. ${ }^{21}$ 
PSYCHE NMR was chosen for this analysis as it is seen to be the most tolerant of strong coupling ${ }^{21}$ as well as being very easy to implement; two real advantages when it comes to the analysis of complex pyrolysis oils.

The simple application of PSYCHE has led to its use in conjunction with other NMR techniques including PSYCHE-iTOCSY (TOtal Correlation SpectroscopY) and PSYCHE-iCOSY (Correlation Spectroscopy). ${ }^{21}$ In this study, we combined PSYCHE with the previously introduced DOSY NMR in a method known as PSYCHE-iDOSY. PSYCHE-iDOSY is a unique pseudo 3D method involving the combination of a diffusion dimension with PSYCHE pure shift. ${ }^{22,23}$

Despite being invented in 2016, PSYCHE-iDOSY has not yet been exploited for the analysis of pyrolysis oils. ${ }^{24}$

Both PSYCHE and DOSY experiments combined have the capability to verify signals from the $1 \mathrm{D} 1 \mathrm{H}$ spectrum as belonging to a specific component, which can then be quantified using software such as Chenomx NMR Suite ${ }^{T M}$. Chenomx works by scanning the ${ }^{1} \mathrm{H}$ NMR spectrum of the sample and matching the combination of peaks with those from a database of known compounds. With a known concentration of an internal standard, 3-trimethylsilyl $\left(2,2,3,3-{ }^{2} \mathrm{H}_{4}\right)$ propionic acid sodium salt (TSP), their relative abundances can also be estimated. This methodology enables the identification of a small library of 'marker' compounds whose combination and concentrations could be specific to each upgraded oil, thus serving as an indicator as to the upgrading process undergone by the oil.

This combined use of PSYCHE-iDOSY and Chenomx NMR Suite ${ }^{T M}$ aimed to provide a simple and improved way to analyse pyrolysis oil; but also serve as an example for potential applications in the analysis of other complex mixtures. 


\section{Materials and methods}

\subsection{General}

Five samples of pyrolysis oil were supplied by Future Blends Ltd., a biofuel production company specialising in pyrolysis and upgrading procedures. The samples consist of one crude and four refined samples (Table 1).

\begin{tabular}{|c|c|}
\hline Pyrolysis Oil & Upgrade Technique \\
\hline Crude & Filtered Pyrolysis Oil \\
\hline Upgrade 1 & Advanced Pyrolysis Oil (APO $^{\mathrm{TM}}$ ) \\
\hline Upgrade 2 & APO $^{\mathrm{TM}}+$ Hydrodeoxygenation (HDO) \\
\hline Upgrade 3 & Volume Reduction (VR) \\
\hline Upgrade 4 & VR + Hydrodeoxygenation (HDO) \\
\hline
\end{tabular}

Table 1. The description provided by Future Blends Ltd for each upgrade.

The removal of the sugar components and retention of aromatic and phenolic based lignin fractions within the pyrolysis oil is used to give APO ${ }^{\mathrm{TM}}$ (Upgrade 2), as detailed by M. Bogarra-Macias ${ }^{25}$.

The pyrolysis oils (10-30mgs) were weighed into separate HPLC vials. A 100mM TSP

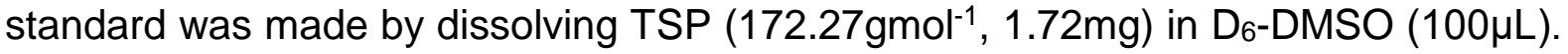
D6-DMSO $(580 \mu \mathrm{L})$ was added to each vial along with $10 \mu \mathrm{L}$ of the TSP solution. The samples were then transferred to $5 \mathrm{~mm}$ NMR tubes for analysis. Sonication was used for samples which did not dissolve easily. The same procedure was used in $3 \mathrm{~mm}$ tubes for DOSY and PSYCHE-iDOSY experiments using only $160 \mu \mathrm{L}$ of $\mathrm{D}_{6}-\mathrm{DMSO}$. The narrower tubes were employed to minimise the convection effects within our samples which could potentially affect the diffusion results. 


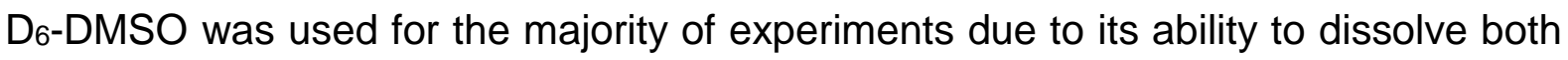
polar and non-polar compounds. Samples were also prepared in $10 \% \mathrm{D}_{2} \mathrm{O} / \mathrm{H}_{2} \mathrm{O}$ in order to be analysed by Chenomx ${ }^{\mathrm{TM}}$ software. This solvent change was required as some chemical shift are particularly solvent dependent, and peak drifting needs to be minimised in order to allow accurate peak matching and identification by Chenomx.

\subsection{Parameters}

A summary of the most important parameters is given in supplementary material S6.

All NMR spectroscopy experiments were conducted on a Bruker Avance III $600 \mathrm{MHz}$ three-channel FT-NMR spectrometer, equipped with a $\mathrm{TXI}{ }^{1} \mathrm{H} /{ }^{2} \mathrm{D}\left\{{ }^{13} \mathrm{C},{ }^{15} \mathrm{~N}\right\}$ probehead. The NMR spectrometer is automated using Bruker IconNMR 5.0.7 and all spectra processed with Bruker TopSpin 3.5.7 as the control software and processing software and Dynamics Center 2.4.9 for DOSY and PSYCHE-iDOSY analysis. Acquisition parameters for standard experiments (1D $1 \mathrm{H}$ and $1 \mathrm{H}$ DOSY), was done with: $\mathrm{P} 1=$ $7 \mu \mathrm{s} ;$ flip angle $=90^{\circ} ; \mathrm{D} 1=10 \mathrm{~s}, \mathrm{NS}=64$. Acquisition parameters for pure shift experiments $1 \mathrm{D} 1 \mathrm{H}$ and $1 \mathrm{H}$ DOSY: $\mathrm{P} 1=7 \mu \mathrm{s}$; flip angle $=90^{\circ}$ hard pulse; $\mathrm{D} 1=1 \mathrm{~s} ; \mathrm{NS}$ $=512 / 32$ respectively. Prior to processing by TopSpin, the $1 \mathrm{D}$ PSYCHE and $2 \mathrm{D}$ PSYCHE-iDOSY spectra needed to be reconstituted respectively from their pseudo 2D and pseudo 3D acquired datasets. This was performed by running the automation program au_pshift_nD.mf as provided in the supplementary information to their original papers by Foroozandeh et al. ${ }^{22}$ Chenomx ${ }^{\mathrm{TM}}$ NMR Suite was also used as an NMR analysis software used for metabolite detection and biomarkers.

The majority of experiments were run in automation apart from the PSYCHE and PSYCHE-iDOSY experiments which were set up manually. Phase adjustment, baseline correction and integration was carried out manually for all experiments. This 
includes manual editing of the slope and bias of integrals as well as calibration of integrals for quantitation.

Inversion Recovery experiments were run initially to identify the T1 relaxation with and without spiking by $\mathrm{Chr}(\mathrm{Ac})_{2}$. These experiments identified the relaxation time as below 4 seconds for the entire sample without $\mathrm{Chr}(\mathrm{Ac})_{2}$ thus standard experiments with D1's of 10 s were sufficient. Samples undergoing pure shift experiments were spiked with $\mathrm{Chr}(\mathrm{Ac})_{2}$ to reduce the required D1 time and allowed for greater signal averaging per unit time.

\section{Results and Discussion}

\subsection{Observations}

Visual comparison of the oils shows that crude pyrolysis oil and upgrades 2 and 4 were viscous but pourable oils, whereas upgrades 1 and 3 are extremely solid and sticky oils. All were soluble in D6-DMSO but upgrades 1, 2 and 4 were less soluble in $\mathrm{D}_{2} \mathrm{O}$. This suggests that the crude oil and upgrade 3 have a higher content of watersoluble compounds.

\subsection{One-Dimensional Proton NMR}

The first approach in the NMR analysis of the oils was to replicate the work done by Hao et al. ${ }^{5} \mathrm{~A}$ one-dimensional solvent-suppressed ${ }^{1} \mathrm{H}$ spectrum was acquired for the initial comparison. A standard NOESY-1D presaturation experiment was used to selectively remove the DMSO peak. Fig. 1 shows the resulting spectra stacked one on top of another,. The first notable observation is that upgrade 1 has the highest concentration of water (water peak is seen at $-3.4 \mathrm{ppm}$ ) and upgrade 2 the lowest. 
Upgrade 3 looked very similar to the crude in the aliphatic region and most other regions. Upgrades 2 and 4 are less concentrated in carboxylic acid and aldehyde environments and upgrade 4 has significantly increased in aliphatic environments.

\section{FIGURE 1}

Due to the complexity of the mixtures, a simple proton spectrum of these oils is very difficult to analyse quantitatively. With extensive overlapping of peaks, identifying single components in the mixture using ${ }^{1} \mathrm{H}$ NMR alone is near impossible. In previous studies, the ${ }^{1} \mathrm{H}$ spectra have been analysed by regions of interest. The same approach was taken with this study and Table 2 shows how these regions have been allocated. A display of the oils regions is given in Fig. 1 (bottom).

\begin{tabular}{|c|c|}
\hline Chemical Shift Region (ppm) & Functionality \\
\hline $0.50-2.50$ & Aliphatic, aliphatic alcohols, allylic, benzylic, alkynyl \\
\hline $2.50-4.40$ & Alcohols, ethers, methoxy, amines \\
\hline $4.40-6.00$ & $\begin{array}{r}\text { Methoxy, vinylic, alkenes, non-conjugated alkenes, } \\
\text { phenols, amines, sugars }\end{array}$ \\
\hline $6.00-8.40$ & $\begin{array}{c}\text { Conjugated and non-conjugated alkenes, aromatics, } \\
\text { phenols, amides, carbohydrates and sugars }\end{array}$ \\
\hline $8.40-10.30$ & Carboxylic acids, aldehydes, amides \\
\hline
\end{tabular}

Table 2. Regions of interest used for integration analysis across all oils

The boundaries of these chemical shift regions are not clearly defined and regional overlap is inevitable. The complex constitution of the oil, as well as the choice of deuterated solvent, often leads to shifting of peaks. For this reason, the following 
analyses have a significant margin of error associated with them. However, this approach of integrating by regions is merely reiterating what has already been carried out in previous literature in order to highlight the limitations of this method.

\subsection{Statistical procedures}

In addition to the error arising from regional overlap, the manual phasing and correction of the bias and slope of each integral introduces operator-derived intrasample variance. To account for this, 1D NMR measurements were done in triplicate, and relative standard deviations were calculated. These results are displayed in Fig 2. Integral regions were set and imported into each spectrum to ensure consistency.

\section{FIGURE 2}

Fig. 2 shows that for each oil, the most concentrated region is the aliphatic region 0.50 $-2.50 \mathrm{ppm}$. The least concentrated region is the carboxylic acids and aldehydes region $8.40-10.30$ and out of all the oils, upgrade 1 is the most concentrated in this region. Upgrades 2 and 4 have substantially increased in aliphatic protons compared with pyrolysis oil and both are also lower in oxygen-containing compounds and alkenes (2.50 - 6.00). Upgrade 3 is very similar to the crude oil in most regions and is actually seen to be higher in concentration in the oxygen-containing compounds.

This method provides a good basis for the comparison of the pyrolysis oils, however, due to the overlap of these regions, these figures are not completely representative of functional group abundance. Also, the observations made from this line of analysis are not detailed enough to be able to distinguish one oil from another.

\subsection{Initial Conclusions}


From the initial analysis using 1D ${ }^{1} \mathrm{H}$ NMR, it appears that upgrades 2 and 4 have undergone the most effective refinement. They both appear to have increased in aliphatic environments and reduced in oxygen-containing and acidic compounds. From ${ }^{1} \mathrm{H}$ NMR analysis thus far, upgrade 3 appears very similar to the crude pyrolysis oil. Upgrade 1 has improved marginally in some regions but overall is not too dissimilar to the crude pyrolysis oil. What is clear, is that it would be difficult to identify and quantify, with any degree of certainty individual components and so 1D 1H NMR lacks the dimensionality on its own to characterise accurately pyrolysis oil/upgrades. Direct application of Chenomx NMR suite fitting to 1D ${ }^{1} \mathrm{H}$ Pyrolysis oil spectra would yield incorrect assignments owing to multiplet overlap and cannot take into account the size of the molecule that yields a set of 1D NMR signals and defaults to the smallest metabolic unit. This is useful in biological fluids, but could create vexatious results when dealing with complex composition products from pyrolysis.

\subsection{Diffusion-Ordered NMR Spectroscopy}

Diffusion-ordered spectroscopy can help in the virtual separation of overlapping peaks by plotting the chemical shift against the diffusion coefficient. Supplementary Information S2 shows the DOSY NMR of the pyrolysis oils.

The DOSY spectra show good separation for the carboxylic acid and aldehyde proton region in crude oil. Upgrades 1 and 3 are very similar to the crude with a vast array of peaks throughout the spectrum, with upgrade 3 having almost identical peaks in the carboxylic acid region. Upgrades 2 and 4 appear completely diminished in this region - in line with the findings from the 1D NMR. 
Upgrade 4 displays how peaks with a very similar chemical shift within the region of 3 -5ppm have been clearly separated into different compounds.

The processing of DOSY spectra requires manual peak picking for analysis in Dynamics Center. Large highly concentrated areas cause some baseline rolling which leads to some smaller peaks becoming masked. For instance, the regions of $0.5-2 \mathrm{ppm}$ and 6-7.5ppm are so concentrated that some small peaks either side of these would have fallen under the peak picking threshold and hence been missed in the data analysis. Baseline rolling can therefore lead to a loss of detail in the DOSY spectrum.

\subsection{Pure Shift NMR Spectroscopy - PSYCHE}

Although the upgrading techniques named $\mathrm{APO}^{\mathrm{TM}}$ and $\mathrm{VR}$ are proprietary, this information helps to explain some of the observations being made. The 1D proton and DOSY NMR conducted so far suggested that upgrades 2 and 4 are greatly reduced in oxygen containing compounds, carboxylic acids/aldehydes and have increased levels of aliphatic protons. Given they have both undergone hydrodeoxygenation (HDO), many of these observed trends can now be explained. There has been much research into the effects of HDO on complex mixtures. ${ }^{26}$

As outlined before, the main limitation with using pyrolysis as a biofuel is the immiscibility with common fuels due to the high concentration of oxygen.

The HDO of phenolics, furans and carboxylic acids have been investigated in previous studies. ${ }^{27,}{ }^{28}$ During the hydrodeoxygenation process, alcohol, ether, ester, carboxylic acid and carbonyl groups are cleaved and hydrogenated along with the saturation of aromatics. ${ }^{29}$ This results in a complex set of reactions ultimately increasing alkane concentration and reducing oxygen content. 
With this known, it is now possible to make more relevant comparisons between the oils. For instance, to compare the $\mathrm{APO}^{\mathrm{TM}}$ and VR procedures which give upgrades 1 and 3 respectively and comment on the relative successes of these.

PSYCHE-iDOSY gives us these fingerprint type spectra (Supplementary Information S3) which are individual to each upgrade. For instance, the crude pyrolysis oil has a large range of molecular weights present and multiple peaks within the carboxylic acid/aldehyde region. It is very concentrated in the aromatic region with some poorly resolved peaks even in the PSYCHE-iDOSY spectra. These acid peaks have been removed in upgrades 1 and 3 and the range of aromatic environments have decreased in both upgrades. The aliphatic region in upgrade 1 is better resolved with some clear peaks being identifiable from the cluster originally in the crude oil.

The range of molecular weights has greatly reduced in upgrades 2 and 4 . There is much less error in the diffusion dimension and peaks are well resolved.

Overall, peaks in the PSYCHE-iDOSY spectra see greatly reduced error in the chemical shift dimension thanks to the elimination of multiplicity. However, when compared to the DOSY spectra, the error in the diffusion dimension appears to have increased with peaks being vertically elongated as seen in Fig. 3 . This indicates that we sacrifice diffusion resolution for chemical shift resolution by moving from DOSY to PSYCHE-iDOSY.

\section{FIGURE 3}

Often, systematic errors in diffusion coefficient arise from spatial non-uniformity of the pulsed field gradients, sample convection or disturbance of the field frequency lock as 
extensively detailed by Kiraly et al. ${ }^{30}$ The use of transverse pulsed field gradients could help to eliminate these sources of error.

It is worthy of note that by taking a slices from the PSYCHE-iDOSY, we were able to visualise the decoupling of multiplets far more clearly than by using the 1D PSYCHE experiment alone (Fig. 4).

\section{FIGURE 4}

This simplification of the pyrolysis oil spectra coupled with the estimation of RMM for components 3.7.6 served to confirm the identity of specific components in the pyrolysis oil and upgrades for their quantification by Chenomx ${ }^{\mathrm{TM}}$.

\subsection{Single Component Detection with Chenomx $x^{T M}$}

\subsubsection{Principles}

The next step in the analysis of these pyrolysis oils was to identify single components within the spectra and attempt to compare their relative concentrations across the upgrades. With the identification of a few compounds of known molecular weights, a calibration curve which related the diffusion coefficient to the molecular mass was constructed. Given that the TSP and DMSO peaks are easily identifiable in each of the PSYCHE-iDOSY spectra, these served as the first two data points. ${ }^{31,32}$ Metabolite detection software, Chenomx ${ }^{\mathrm{TM}}$, was used to provisionally identify other compounds present in the oils. The software is aimed at the analysis of complex mixtures and works by matching the patterns of peaks of known substances with those found in the ${ }^{1} \mathrm{H}$ spectra of the sample. Given that TSP is present in all samples at a known concentration, Chenom $x^{\mathrm{TM}}$ can approximate the concentration of any 
successfully identified compounds. Due to the primary function of the software as a metabolite detection aid and its intended use for food, blood plasma, cell culture mixtures etc., the samples must be in an aqueous medium. NMR samples of the oils were therefore prepared in $\mathrm{D}_{2} \mathrm{O}$ for this section of the analysis. It is also important to note that this method of component assignment is unproven in pyrolysis oils, hence the use of PSYCHE and DOSY experiments to confirm the Chenomx ${ }^{\mathrm{TM}}$ assignment. Supplementary information S4, for example shows the four peaks of highest intensity in upgrade 4 , appearing at $\delta 0.9 p p m, 1.35 p p m, 1.42 p p m$ and $3.41 p p m$ with relative integrals of 3:2:2:2 respectively. Chenomx identified these peaks as valerate (at $\sim 100 \mathrm{mM}$ ) by matching three out of these four peaks as shown in Fig. 5 .

\section{FIGURE 5}

The triplet arising from the $\mathrm{CH}_{2}$ group adjacent to the carboxylate was predicted to appear at 2.31ppm (Fig. 5), however, on further inspection it was found shifted downfield at 3.41ppm. This led to the suggestion that this $\mathrm{CH}_{2}$ group lay closer to an

electronegative atom, for instance, the hydroxyl group on C1 of 1-butanol (Supplementary Information, S5). The predicted spectrum of 1-butanol was found to show that indeed these protons do appear downfield at 3.3ppm.

In an attempt to validate this proposal, a search for these peaks in the PSYCHEiDOSY spectrum of upgrade 4 concluded that these four peaks had very similar diffusion coefficients; implying they belong to the same compound (Fig. 6). 
They have a larger diffusion coefficient than TSP and DMSO but smaller than ethylene glycol which, given the RMM's of these compounds and their corresponding order of diffusion coefficients supports the positive identification of butanol.

\section{FIGURE 6}

\subsubsection{Calibration Curve for RMM Determination}

Relative molecular mass (RMM) prediction of compounds was achieved through the method introduced by Li et al ${ }^{33}$, which allows for prediction of RMM by the use of internal references where small molecule prediction has been shown to give excellent results. If viscosity change of the solutions and density changes are kept to a minimum we can accurately predict the RMM and derive information on the solution state, aggregation number and improve the interpretation of reaction pathways.

Upgrade 4 has four known compounds present, TSP, DMSO, butanol and ethylene glycol (Supplementary materials (S7) shows corresponding molecular masses and diffusion coefficients of these). These peaks were identified in the DOSY spectrum and a calibration curve was constructed which compared diffusion coefficient with molecular weight as shown in the supplementary material (S6).

This calibration curve now allows for the identification of the molecular mass of compounds giving rise to unidentified peaks.

The $\mathrm{R}^{2}$ value of 0.904 represents a satisfactory standard deviation of data points from the trendline, however, the error in its linear fitting can be reduced by increasing the number of data points. Given that the following eight compounds (Table 3 ) have been identified in crude pyrolysis oil, a much more accurate calibration curve should be able 
to enhance analysis. However, they first need to be identified in the $1 \mathrm{D}{ }^{1} \mathrm{H}$ spectrum, and subsequently in the DOSY spectrum. 


\begin{tabular}{|c|c|c|c|}
\hline Compound & $\begin{array}{c}\text { RMM } \\
\left(\mathbf{g m o l}^{-1}\right)\end{array}$ & 1H NMR d (ppm) & $\begin{array}{c}\text { Diffusion } \\
\left.\text { Coefficient } \mathbf{~ ( m}^{\mathbf{2}} \mathbf{s}^{-\mathbf{1}}\right)\end{array}$ \\
\hline Methanol & 32.04 & 3.34 & \\
\hline Ethylene Glycol & 62.07 & 3.65 & $6.48 \times 10^{-10}$ \\
\hline Hydroxyacetone & 74.08 & $2.13,4.35$ & $4.40 \times 10^{-10}$ \\
\hline Butanol & 74.12 & $0.87,1.39,1.67,3.30$ & $3.56 \times 10^{-10}$ \\
\hline DMSO & 78.13 & 2.55 & $2.38 \times 10^{-10}$ \\
\hline Catechol & 110.10 & $6.83,6.91$ & \\
\hline TSP & 127.27 & 0.00 & \\
\hline Levoglucosan & 162.14 & $3.51,3.67,3.74,4.07,4.61,5.43$ & \\
\hline
\end{tabular}

Table 3: The 8 compounds successfully identified in pyrolysis oil by Chenomx, their molecular mass and PSYCHE diffusion coefficients.

On this basis characteristic compounds and their relative concentrations could be identified in each of the oils; these could potentially act as indicators as to which upgrading process has taken place for an unknown pyrolysis oil. For instance, if upgrades 2 and 4 show complete loss of a certain compound which is present in all other oils, this could be accounted for by the HDO process. If an unknown sample of oil was analysed and found to be depleted in that substance, it could suggest it had also undergone HDO.

\subsubsection{Hydroxyacetone}

The most common compound in the raw pyrolysis oil was found to be hydroxyacetone at $\sim 70 \mathrm{mM}$. It is a common metabolite of many sugars with both a carbonyl and an alcohol group making it water soluble and polar. For this reason, we can expect the 
HDO to effectively eliminate this compound from upgrades 2 and 4. Fig. 5 shows how the theoretical peaks are matched to those found in the pyrolysis oil. The relative concentrations of this compound in all samples is displayed in fig. 7.

3.7.3 The five indicator compounds chosen for pyrolysis oil characterisation Using the same peak matching methodology as for hydroxyacetone, the concentrations of four additional compounds were estimated in crude oil and four upgraded oil samples.

\section{FIGURE 7}

From figure 7 we can see that upgrade 1 has the lowest levels of all five compounds. Following this, upgrade 2 is just as depleted in hydroxyacetone and levoglucosan, however levels of ethylene glycol, methanol and catechol were, in fact, higher than those seen in upgrade 1 . This indicates the HDO of upgrade 2 results in an increase in methanol and catechol, i.e. they are by-products of the hydrogenation or deoxygenation of other compounds present in upgrade 1 . Upgrade 3 is the most concentrated in three of the five indicators amongst the upgraded oils, whilst upgrade 2 is the highest in methanol and catechol. Upgrade 4 is always lower in concentration than upgrade 3 in all five compounds. This suggests that the hydrodeoxygenation of upgrade 3 removes the majority of these compounds. The APO ${ }^{\mathrm{TM}}$ refinement to give upgrade 1 has notably reduced the concentration of hydroxyacetone and the VR upgrading process leading to upgrade 3 is not as efficient at reducing hydroxyacetone as the APO ${ }^{\mathrm{TM}}$ process. As expected, upgrades 2 and 4 have low levels of hydroxyacetone. 


\subsubsection{Limitations of Chenomx ${ }^{\mathrm{TM}}$}

Although the above observations are extremely usefully when it came to the characterisation of these oils, there are many limitations associated with this method of analysis. Firstly, Chenomx only works with aqueous solutions; meaning that the oil samples can only be analysed for the water-soluble compounds and the information concerning the hydrophobic components is in fact lost.

Secondly, although concentrations appeared relatively high in the $\mathrm{D}_{2} \mathrm{O}$ samples, identification of the same peaks in the corresponding DMSO samples was difficult; with many peaks being lost in the noise and/or shifted from the frequency range where they were found in aqueous media. This rendered their identification within the DOSY spectra difficult.

Chenom $\mathrm{x}^{\mathrm{TM}}$ has a detailed database of compounds $(>300)$ and functions well in its intended use as a metabolite detector, detecting sugars and alcohols without any problem. However, Chenomx ${ }^{\mathrm{TM}}$ doesn't detect many compounds which are expected to be present in pyrolysis oils; such as lignins and other long chain hydrocarbons. This presented the greatest limitation, as some of the most intense peaks present were unidentifiable with Chenomx. This was illustrated by the false attribution of peaks to valerate; which was correctly attributed to butanol, a known concentrated compound in pyrolysis oils and fuels.

\section{Conclusion}

Given the difficulty of analysing pyrolysis oils by a large range of analytical techniques such as MS, HPLC and GCMS, the use of NMR methodologies has proven to be very valuable, however, the majority of NMR analysis of pyrolysis oil until this point has focused on identifying general classes of components. In this paper, we report, for the first time, the use of PSYCHE and PSYCHE-iDOSY experiments to verify signals for 
components that could then be quantified with Chenomx ${ }^{\mathrm{TM}}$ Software. Proton NMR provided a reasonable overview of the oils composition and allowed for the comparison of oils by regions of interest. Given that the oxygen content of the oils is the main limitation with fuel miscibility, PSYCHE-iDOSY enabled the simplification of the NMR spectrum and validation of 5 oxygen containing components, provisionally assigned by Chenomx ${ }^{\mathrm{TM}}$ despite the limitations of Chenomx ${ }^{\mathrm{TM}}$ as a metabolite identifier. Whilst, PSYCHE-iDOSY NMR is a relatively new technique and has never been implemented in the analysis of pyrolysis oils before, the data collected during this study sets a precedent for the establishment of a PSYCHE database for complex mixtures to better enable the assignment of components in unknown samples. ${ }^{34}$ This technique, in tandem with the building of a database of fuels like Chenom ${ }^{\mathrm{TM}}$ would allow attribution of compounds through separation of structurally similar compounds by their RMM and chemical shifts. With the combination of certain statistical analytical methods, PSYCHE-iDOSY NMR shows great potential in the analysis of complex mixtures and by simplifying ${ }^{1} \mathrm{H}$ spectra using PSYCHE and by adding the additional dimension of diffusion and estimation of RMM, work is currently underway to combine these techniques to afford a multivariate (PCA) map to group pyrolysis oil upgrades by the type of upgrading procedure and also the duration of said treatment. This would potentially enable a clear, unambiguous, non-destructive fingerprinting of different pyrolysis oil/upgrade types, without the need for protracted, costly separation techniques.

\section{Acknowledgements}

The authors are grateful to Kingston University for their support for this project. 


\section{Conflict of Interest}

This research did not receive any specific grant from funding agencies in the public, commercial, or not-for-profit sectors. The authors declare no conflict of interest.

\section{Glossary}

$\mathrm{APO}^{\mathrm{TM}}=$ Advanced Pyrolysis Oil

BASH = Band Selective homodecoupling

BIRD = Bilinear Rotation Decoupling

COSY = Correlation Spectroscopy

$\operatorname{Cr}(\mathrm{AcAc})_{3}=$ Chromium Acetylacetonate

$\mathrm{D}_{2} \mathrm{O}=$ Deuterated Water

D6-DMSO = Deuterated Dimethyl Sulfoxide-d6

DOSY = Diffusion Ordered Spectroscopy

GCMS = Gas Chromatography - Mass Spectrometry

HDO = Hydrodeoxygenation

HPLC = High Performance Liquid Chromatography

HSQC = Heteronuclear Single Quantum Correlation

NMR = Nuclear Magnetic Resonance

NOE $=$ Nuclear Overhauser Effect

NOESY = Nuclear Overhauser Effect Spectroscopy

NUS = Non-Uniform Sampling

PCA = Principle Component Analysis

PSYCHE = Pure Shift Yielded by Chirp Excitation 
$\mathrm{RMM}=$ Relative Molecular Mass

$\mathrm{S} / \mathrm{N}=$ Signal-to-Noise

TOCSY $=$ Total Correlation Spectroscopy

TSP = Trimethylsilylpropanoic Acid

VR = Volume Reduction

\section{References}

1. Czernik S, Bridgwater A. V. Overview of Applications of Biomass Fast Pyrolysis Oil. Energy\&fuels. 2015; 29 (4) 2471-2484

2. Yang H, Yao J, Chen G, Ma W, Yan B, Qi Y. Overview of Upgrading of Pyrolysis Oil of Biomass. Energy Procedia. 2014; 61 1306-1309.

3. Mohan D, Pittman C. U, Jr, Steele P. H. Pyrolysis of Wood/Biomass for Bio-oil: A Critical Review.Energy\&fuels. 2006; 20 (3) 848-889

4. Barnés C, de Visser M.M, van Rossum G, Kersten S.R.A, Lange J.-P. Liquefaction of wood and its model components. Journal of Analytical and Applied Pyrolysis. 2017; 125 136-143

5. Hao N, Ben H, Yoo C. G, Adhikari S, Ragauskas A. J. Review of NMR Characterization of Pyrolysis Oils.Energy\&fuels. 2016; 30 (9) 6863-6880

6. Baird C, and Cann M. Environmental Chemistry. 5thed. New York: W. H. Freeman and Company; 2012

7. Biswas B, Pandey N, Bisht Y, Singh R, Kumar J, Bhaskar T. Pyrolysis of agricultural biomass residues: Comparative study of corn cob, wheat straw, rice straw and rice husk. Bioresource Technology. 2017; 237 57-63

8. Huber G. W, Iborra S, Corma A. Synthesis of Transportation Fuels from Biomass: Chemistry, Catalysts and Engineering. Chemical Reviews. 2006; 106 (9) 4044-4098

9. Qiang L, Wen-Zhi L, Zhu, Xi-Feng. Overview of fuel properties of biomass fast pyrolysis oils. Energy Conversion and Management. 2009; 50 (5) 1376-1383

10. Vispute, Tushar, Pyrolysis oils: characterization, stability analysis, and catalytic upgrading to fuels and chemicals. 2011

11. Talmadge M. S, Baldwin R. M, Biddy M. J, McCormick R. L, Beckham G. T, Ferguson G.A et al. A perspective on oxygenated species in the refinery integration 
of pyrolysis oil. Green Chemistry. 2014; 16 (2) 407-453

12. Bridgwater A.V. Renewable fuels and chemicals by thermal processing of biomass. Chemical Engineering Journal. 2003; 91 (2) 87-102

13. Sharifzadeh M, Richard CJ, Liu K, Hellgardt K, Chadwick D, Shah N. An integrated process for biomass pyrolysis oil upgrading: A synergistic approach. Biomass and Bioenergy. 2015; 76 108-117

14. Ben H, Ragauskas A. J. NMR Characterization of Pyrolysis Oils from Kraft Lignin. Energy \& fuels. 2011; 25 (5) 2322-2332

15. Castañar L. Pure shift $1 \mathrm{H}$ NMR: what is next? Magnetic Resonance in Chemistry. 2017; 55 (1) 47-53

16. Anderson W. A, Freeman R. Influence of a Second Radiofrequency field on HighResolution Nuclear Magnetic Resonance Spectra.The Journal of Chemical Physics. 1962; 3785

17. Meyer NH, Zangger K. Simplifying Proton NMR Spectra by Instant Homonuclear Broadband Decoupling.AngewandteChemie International Edition. 2013; 52 (28) 7143-7146.

18. Garbow J. R, Weitekamp D.P, Pines A. Bilinear Rotation Decoupling of Homonuclear Scalar Interactions. Chemical Physics Letters. 1982; 93 (5) 504-509

19. Zangger K. Pure shift NMR. Progress in Nuclear Magnetic Resonance Spectroscopy. 2015; 86-87 1-20

20. Castañar L, Nolis P, Virgili A, Parella T. Full Sensitivity and Enhanced Resolution in Homodecoupled Band-Selective NMR Experiments. Chemistry - A European Journal. 2013; 19 (51) 17283-17286.

21. Castañar L, Parella T. Broadband 1H Homodecoupled NMR Experiments: Recent Developments, Methods and Applications. Magnetic Resonance in Chemistry. 2015; 53 (6) 399-426.

22. Foroozandeh M, Castañar L, Martins L. G, Sinnaeve D, Poggetto G. D, Tormena C. F, et al. Ultrahigh-Resolution Diffusion-Ordered spectroscopy. Angewandte Chemie International Edition. 2016; 55 (50) 15579-15582.

23. Nilsson M, Morris GA. Pure Shift Proton DOSY: Diffusion-Ordered $1 \mathrm{H}$ Spectra Without Multiplet Structure. Chemical Communications. 2007; (9) 933

24. Facey G, Double Presaturation, University of Ottawa NMR Facility Blog. 2012; Available from:

http://u-of-o-nmr facility.blogspot.co.uk/2012/03/doublepresaturation.html [Accessed $11^{\text {th }}$ December 2017] 
25. Bogarra-Macias M, Doustdar O, Fayad MA, Wyszyński ML, TSOLAkiS A, Ding P, Pacek A, MARTIN P, Overend R, O'Leary S. Performance of a drop-in biofuel emulsion on a single-cylinder research diesel engine. Combustion Engines. 2016;55.

26. Bu Q, Lei H, Zacher AH, Wang L, Ren S, Liang J, et al. A Review of Catalytic Hydrodeoxygenation of Lignin-Derived Phenols from Biomass Pyrolysis. Bioresource Technology. 2012; 124 470-477

27. Lee $\mathrm{H}$, Kim H, Yu MJ, Ko CH, Jeon J-K, Jae J, et al. Catalytic Hydrodeoxygenation of Bio-oil Model Compounds over Pt/HY Catalyst. Sci Rep. 2016; 628765

28. Routray K, Barnett K. J, Huber G. W. Hydrodeoxygenation of Pyrolysis Oils. Energy Technology. 2017; 5 (1) 80-93

29. Su-Ping Z. Study of Hydrodeoxygenation of Bio-Oil from the Fast Pyrolysis of Biomass. Energy Sources. 2003; 25 (1) 57-65

30. Kiraly P, Swan I, Nilsson M, Morris G. A. Improving Accuracy in DOSY and Diffusion Measurements Using Triaxial Field Gradients. Journal of Magnetic Resonance. 2016; 270 24-40

31. Lakshmanan C. M, Hoelscher H. E. Production of Levoglucosan by Pyrolysis of Carbohydrates.Pyrolysis in hot Inert Gas Stream. Industrial and Engineering Chemistry Product Research and Development. 1970; 9 (1) 57-59

32. Foroozaneh M, Adams R. W, Nilsson M, Morris G. A. Ultrahigh Resolution Total Correlation NMR Spectroscopy. Journal of the American Chemical Society. 2014; 136 (34) 11867-11869

33. Li D, Kagan G, Hopson R, Williard PG. Formula weight prediction by internal reference diffusion-ordered NMR spectroscopy (DOSY). Journal of the American Chemical Society. 2009 Mar 26;131(15):5627-34

34. Facey G, Non-Uniform Sampling (NUS), University of Ottawa NMR Facility Blog. 2012; Available from: http://u-of-o-nmr-facility.blogspot.co.uk/2016/05/nonuniformsampling-nus.html [Accessed 22nd February 2018]

\section{Figure Captions}


Figure 1. Comparison of all samples ${ }^{1} H$ NMR spectra. (a) crude pyrolysis oil (b) upgrade 1 (c) upgrade 2 (d) upgrade 3 (e) upgrade 4. Regions of interest highlighted below spectra.

Figure 2: The comparison between the concentration of protons within regions of interest with relative standard deviation. From left to right: Crude oil, upgrade 1, upgrade 2, upgrade 3, upgrade 4.

Figure 3: Comparison of the DOSY (top) and PSYCHE-iDOSY (bottom) of crude pyrolysis oil.

Figure 4. 1D slices taken from PSYCHE-iDOSY experiments for Upgrades 1 (a) and $2(b)$.

Figure 5 .Valerate ion was detected by Chenomx according to these three peaks at 0.89ppm, 1.33ppm and 1.51ppm; later identified as butanol

Figure 6: PSYCHE-iDOSY spectrum of upgrade 4 identifying four known compounds with the $1{ }^{1} H$ PSYCHE spectra above. 
Figure 7: Diagram showing the concentration of five indicator compounds in crude oil (orange), upgrade 1 (purple), upgrade 2 (yellow), upgrade 3 (blue), upgrade 4 (green). 